\title{
Formación de mediadores escolares en territorios de conflicto armado: el caso de la Escuela Normal Superior de La Cruz-Nariño
}

\author{
Training of school mediators in areas affected by armed conflict: the case of Escuela \\ Normal Superior in La Cruz-Nariño
}

\section{Formação de mediadores escolares em territórios de conflito armado: o caso da Escuela Normal Superior de La Cruz-Nariño}

\author{
Juan Pablo Rosero Gomajoa * \\ https://orcid.org/0000-0002-8979-2520 Universidad de Nariño, Pasto, Colombia
}

- Fecha de recepción: 2020-07-28

- Fecha concepto de evaluación: 2020-09-20

- Fecha de aprobación: 2020-09-28 https://doi.org//0.22335/rlct.v|3il.1315

\begin{abstract}
Para citar este artículo / To reference this article / Para citar este artigo: Rosero Gomajoa, J. P. (202I). Formación de mediadores escolares en territorios de conflicto armado: el caso de la Escuela Normal Superior de La Cruz-Nariño. Revista Logos Ciencia \& Tecnología, 13(I), 80-95. https://doi.org//0.22335/rlct.v13il.1315
\end{abstract}

\section{RESUMEN}

El presente estudio, realizado en el año 2019, tuvo como objetivo establecer lineamientos para la formación de mediadores en resolución de conflictos escolares en zonas de conflicto armado. Se llevó a cabo en la Escuela Normal Superior de Mayo, ubicada en el municipio de La Cruz, departamento de Nariño (Colombia), con el cuarto semestre del ciclo complementario (normalistas), compuesto por 35 estudiantes, entre ellos 17 hombres y 18 mujeres, que oscilan entre los 18 y 24 años de edad. La investigación estuvo orientada bajo un paradigma cualitativo, de tipo etnográfico, en el cual se aplicaron entrevistas, encuestas y talleres con miembros de la comunidad educativa, las cuales fueron procesadas por el software Atlas.ti 8. Los resultados versan sobre la necesidad de contemplar la mediación escolar como eje estructural del ejercicio educativo en contextos de conflicto armado. Las conclusiones dejan en evidencia que el conocimiento de herramientas de mediación escolar, por parte de los normalistas, les permite solucionar de manera pacífica y eficaz, los conflictos escolares, y a la vez, posibilitar una convivencia más amena coadyuvando a la reconstrucción del tejido social.

Palabras clave: mediadores escolares, convivencia escolar, educación para la paz, conflicto armado 


\section{ABSTRACT}

The present study, carried out in 2019, aimed to establish guidelines for the training of mediators in the resolution of school conflicts in armed conflict areas. It was carried out in Escuela Normal Superior de Mayo, located in the municipality of La Cruz, Nariño department (Colombia), with 35 students in the fourth semester of an early childhood educator qualification (normalistas), made up of 17 men and 18 women, ranging from 18 to 24 years of age. The qualitative, ethnographic research methodology applied interviews, surveys and workshops with members of the educational community, which were processed using the Atlas.ti 8 software. The conclusions show that providing teachers with knowledge of school mediation tools allows them to solve school conflicts peacefully and efficiently, and at the same time, fosters a more pleasant environment that contributes to coexistence and the reconstruction of the social fabric.

Keywords: school mediators, coexistence in school, education for peace, armed conflict

\section{RESUMO}

Este estudo, realizado no ano de 2019 , teve como objetivo estabelecer diretrizes para a for-mação de mediadores em resolução de conflitos escolares em zonas de conflito armado. Realizou-se na Escuela Normal Superior de Mayo, situada no município de La Cruz, departamento de Nariño (Colômbia), com o quarto período do ciclo complementário (normalistas), composto por 35 estudantes, entre eles 17 homens e 18 mulheres, que oscilam entre os 18 e 24 anos de idade. A pesquisa estava orientada sob um paradigma qualitativo, de tipo etnográfico, no qual se aplicaram entrevistas, enquetes e oficinas com membros da comunidade educativa, as quais foram processadas pelo software Atlas.ti 8 . Os resultados versam sobre a necessidade de contemplar a mediação escolar como eixo estrutural do exercício educativo em contextos de conflito armado.As conclusões evidenciam que o conhecimento de ferramentas de mediação escolar, por parte dos normalistas, lhes permite solucionar de maneira pacífica e eficaz, os conflitos escolares, e, por sua vez, possibilitar uma convivência mais amena, coadjuvando à reconstrução do tecido social.

Palavras-chave: : mediadores escolares, convivência escolar, educação para a paz, conflito armado

Pardo (2019) afirma que los derechos a la vida y la paz son propios de la escuela como institución social, máxime cuando estos han sido vulnerados y degradados por un conflicto armado de más de 50 años en el territorio colombiano (2019). Sumado a esto, problemáticas sociales como el pandillismo, la drogadicción, el microtráfico, la violencia intrafamiliar, la violencia generalizada, entre otras, se insertan y se expanden en el escenario educativo (Chaux, 20I2), dando paso a la denominada violencia escolar y configurando un círculo de situaciones conflictivas en todo el entramado social.

Así, entonces, la violencia en la escuela, denominada también violencia escolar, agresión escolar o bullying (Ortega, 20I0), requiere una atención urgente, más aún, cuando existen cifras alarmantes en relación con dicho fenómeno, tales como las presentadas por la Organización de las Naciones Unidas (2019) en un informe titulado Behind the numbers: Ending school violence and bullying, en el cual se afirma que, a nivel mundial, uno de cada tres estudiantes ha sido acosado por sus compañeros en la escuela al menos una vez al mes. En todas las regiones, el acoso escolar físico es la representación más común dentro de los tipos de intimidaciones, seguido por el acoso sexual, a excepción de Europa y Norteamérica, en donde el acoso psicológico presenta una mayor tendencia.

Aunado a lo anterior, la Organización de las Naciones Unidas para la Educación, Ciencia y Cultura (Unesco, 20I8) advierte que: (I) la tercera parte de los jóvenes del mundo sufre acoso en la escuela, (2) los niños padecen de índices ligeramente superiores de acoso que las niñas, pero en los países donde el acoso es más frecuente, las niñas son más vulnerables y (3) la condición de inferioridad socioeconómica, es el principal indicador para predecir si un joven padecerá acoso en la escuela. 
El panorama se agrava en Latinoamérica, y específicamente en Colombia, puesto que según datos del Ministerio de Educación Nacional (MEN), a pesar de que desde el año 2012 se ha reportado una reducción de la agresión escolar en las instituciones del país, la cifra de menores que reportan ser víctimas de matoneo sigue siendo superior al $20 \%$ a nivel nacional (20I5a). "De acuerdo con un estudio realizado por la Organización para la Cooperación y el Desarrollo Económicos, en Colombia el $7.6 \%$ de los estudiantes aseguró estar expuesto diariamente a algún tipo de maltrato físico en su colegio" (Vivias, 2018).

Esta situación, aparte de aumentar los índices de deserción estudiantil, también genera procesos negativos en la calidad educativa, pues un ambiente hostil en el contexto de enseñanza-aprendizaje perjudica sustancialmente al estudiante en su proceso educativo. Además, el contexto violento repercute en el ejercicio de una ciudadanía sana, con valores solidarios y éticos que permitan la convivencia pacífica de una sociedad (Margolin \& Ceballos, 2004).

Por ello, "es imperativo que las instituciones educativas consoliden procesos pedagógicos, especialmente en la presente coyuntura del posacuerdo que contribuyan efectivamente a generar una interacción humana menos violenta" (Mejía, 2018, p. 5) para que sea el inicio de un proceso de construcción de paz. En este orden de ideas, no cabe duda de que la estrategia educativa para el tratamiento de conflictos escolares, que a la vez son sociales, se convierte en un elemento clave para iniciar el proceso hacia la generación de una cultura democrática y pacífica (Santiesteban, 2004).

Frente a lo descrito, se torna necesaria la formación docente, especialmente normalistas, con relación a la mediación del conflicto escolar, toda vez que son los profesores los primeros en ser llamados a dar una alternativa de solución y orden cuando un evento de violencia, discrepancia o alteración, sucede en el recinto escolar. En este sentido, se vuelve pertinente el establecimiento de lineamientos para la formación de mediadores en resolución de conflictos escolares y de especial importancia si estos actúan en contextos de conflicto armado, como en el caso de la Escuela Normal Superior del Mayo, en La Cruz, Nariño.

Una aproximación a la caracterización del municipio de La Cruz

El departamento de Nariño, ubicado al suroccidente colombiano, cuenta con una división regional categorizada en 13 subregiones. Entre estas se encuentra la subregión del Río Mayo, la cual comprende los municipios de Belén, Colón, El Tablón de Gómez, San Bernardo, San José de Albán, San Pablo y La Cruz, este último localizado al nororiente del departamento, a una distancia de 103 kilómetros de la ciudad capital, Pasto.

El municipio de La Cruz, según autoridades municipales, está compuesto por 18220 habitantes, de los cuales, el $63.72 \%$ se localizan en el sector rural y el $36.28 \%$, en la cabecera municipal (Alcaldía Municipal de La Cruz, 2020). La actividad económica dominante en el casco urbano, es la comercialización de productos derivados de actividades agrícolas y pecuarias, a su vez, el sector rural se caracteriza por el desarrollo de una economía campesina, con cultivos de pancoger e intercambio de bienes de subsistencia (Quintero, 2019).

Respecto al tema del conflicto armado, estudios afirman que

para el año 2015 se encontraban registradas 2244 personas en el Registro Único de Víctimas (RUV) de la unidad para la atención y reparación integral a las víctimas del conflicto armado en Colombia, lo cual equivale al $12.31 \%$ de la población total del municipio. (Quintero, 2019, p. 42)

Cabe señalar que el hecho victimizante más frecuente es el desplazamiento forzado, con un $73 \%$ del total de los hechos presentados, seguido por el homicidio $y$, por último, el despojo de tierras y bienes muebles (Alcaldía Municipal de La Cruz, 2016).

Es importante señalar que el municipio de La Cruz, al encontrarse geográficamente ubicado en un corredor estratégico, que permite la fácil comunicación entre los departamentos de Nariño, Cauca y Putumayo, ha sido apropiado por grupos armados al margen de la ley, que han expandido sus dominios territoriales para el tráfico de armas y para la producción y comercialización de cultivos ilícitos como la coca y la amapola. Esto ha caracterizado a La Cruz como un escenario de múltiples actos de violencia a causa del conflicto armado y el narcotráfico, que se ha presentado en el macizo colombiano, el cual se convierte en una de las regiones con uno de los más altos índices de violencia en el país. (Quintero, 2019, p. 44)

Estudios históricos sitúan en 199/ el inicio de la presencia de grupos subversivos como las Fuerzas Armadas Revolucionarias de Colombia (FARC-EP), en el municipio, toda 
vez que estos, en el afán de una exploración y expansión de territorios para sus actividades beligerantes, incursionan la zona de la hoy conocida subregión del Río Mayo (Bastidas \& Ordóñez, 2004). Es al finalizar la década de los noventa cuando comienzan a desencadenarse los sucesivos ataques a la población civil, en una lucha por el dominio territorial del municipio de La Cruz, "generando una serie de secuelas en la población civil como el desplazamiento forzado, la destrucción de sus bienes y la muerte de múltiples personas que habitaban este territorio" (Quintero, 2019, p. 46).

Aunado a lo anterior, cuando se analiza el índice de vulnerabilidad de las regiones, el cual mide variables como la violencia, la demografía, el capital humano, la concentración de la propiedad, la capacidad económica, entre otras (PNUD, 20II), se encuentra que la subregión del Río Mayo, presenta un alto índice de vulnerabilidad.

Esta dinámica permite caracterizar a esta subregión del departamento como un territorio con altos niveles de violencia, a raíz del conflicto armado y la existencia de cultivos ilícitos que han confluido en esta subregión. Así mismo, se logra establecer una baja capacidad económica entre su población y un bajo capital humano, configurado a partir de las escasas oportunidades educativas y laborales con las que cuentan los habitantes del municipio, además de los impactos naturales que ha ocasionado la deforestación y la expansión de cultivos ilícitos, lo que convierte a esta subregión en una de las más vulnerables del departamento. (Quintero, 2019, p. 47)

En este contexto, se encuentra sumergida la Escuela Normal Superior del Mayo, la cual ofrece los servicios educativos desde prescolar, primaria y bachillerato, hasta el ciclo complementario. Estos últimos, son docentes en formación que, en su mayoría, se desempeñan en escuelas rurales de Colombia.

\section{Estudios sobre la formación de mediadores en resolución de conflictos escolares}

Es menester mencionar que la figura de un tercero para la resolución, mediación u optimización del conflicto es una idea que ha permanecido a lo largo del tiempo y se ha mantenido en escenarios locales, comunitarios, institucionales, políticos, armados, hasta internacionales (Fisas, 1998). En este sentido, la mediación de los conflictos se constituye como piedra angular de lo que Lederach (1992; 2000) llamaría una paz positiva.
Frente a este postulado se han desarrollado múltiples estudios que abordan la mediación de conflictos escolares, tanto de una manera teórica como práctica. Así entonces, a nivel internacional, el estudio de Fernández y Galán (2010), en el que a través de un proyecto pedagógico intervinieron centros educativos y con un grupo de profesionales en psicopedagogía del municipio de Guadarrama, Madrid (España), implementaron la formación de mediadores escolares en las instituciones educativas de primaria y comprobaron que bajo un trabajo mancomunado entre escuelas, instituciones estatales y familias, podían formar mediadores escolares para fomentar en los estudiantes una convivencia más pacífica, una cultura de paz con mayores cimientos y un crecimiento positivo de la comunidad educativa desde el conflicto.

Bajo esa misma perspectiva, el estudio de lbarrola e Iriarte (2013), aplicado a instituciones educativas de la provincia de Navarra (España), demuestra que la formación de docentes en herramientas de mediación escolar mejora la convivencia del centro educativo, además de que el profesorado percibe mejoras personales en pensamiento reflexivo y empatía en sí mismos. Concluyen que la capacitación en resolución de conflictos escolares coadyuva a afrontar situaciones diarias presentes en el aula y construir una convivencia más sana y pacífica.

En el mismo sentido, estudios desarrollados tanto en Ecuador, por parte de Pérez et al. (2016) y el realizado en República Dominicana por Salcedo y Jennings (2016), apuntan a la formación de mediadores como una herramienta imperativa en los procesos de construcción de una cultura de paz. Afirman que dichos procesos deben abordarse holísticamente, acudiendo al contexto social, cultural, político y económico, para lograr un mayor entendimiento de las causas y consecuencias de los conflictos que se presentan dentro de los claustros educativos.

En Colombia, el estudio realizado por Guajardo (2010) devela la necesidad que tienen los docentes en formación, con relación al aprendizaje en resolución de conflictos, puesto que, los programas curriculares de las licenciaturas tienen un gran déficit en la preparación del tema. Concluye que el profesor capacitado en resolución de conflictos, desde la base de su formación, podrá colaborar en que la convivencia dentro de la institución donde se desempeñe sea la mejor posible.

Asimismo, Luna (2018) señala que la mediación escolar es una estrategia efectiva en el manejo de los conflictos en 
los planteles educativos, y que su impacto es positivo en tanto las instituciones asuman proyectos serios y a largo plazo enmarcados en la construcción de una convivencia sana dentro de la comunidad educativa. Al unísono, el estudio de Martínez (2018) propuso un plan de formación en convivencia que ayude a la transformación de conductas agresivas en oportunidades de aprendizaje. Concluyó que los proyectos de mejoramiento de la convivencia en los centros educativos deben tener como eje transversal la formación de mediadores escolares, en los cuales, a través de ejercicios artísticos, deportivos, lúdicos y democráticos, se construya, en conjunto con toda la comunidad educativa, una cultura de paz.

A su vez, es de vital importancia el estudio de Fiorino et al. (2018), que desde una revisión teórica de distintas formas de resolución de conflictos en el marco de contextos educativos, resaltan la importancia de establecer procesos prácticos en la materia, tal como el trabajo llevado a cabo por la Red Internacional de Formación de Mediadores Xesús R. Jares, impulsado por la Unesco, la cual es una red encargada de la formación de estudiantes, docentes y asociaciones de padres de familia, para que sean los encargados del mejoramiento de la convivencia escolar.

A nivel regional en Colombia, se encuentra como referente el estudio realizado por Ortega (2012), en el cual confiere que los Mecanismos Alternativos de Solución de Conflicto (MASC) son el pilar de la construcción de una sana convivencia, pues dichos mecanismos otorgan las herramientas necesarias para dar una pronta solución a la conflictividad diaria de las escuelas. En concordancia con Ortega (20I2), la investigación de Benavides (2013) hace hincapié en que los MASC, se convierten en esa alternativa pedagógica que ayuda a los establecimientos de enseñanza, a lidiar con problemas como el pandillismo, la drogadicción y demás problemáticas que se extrapolan al escenario educativo.

Aunado a lo anterior, el trabajo de Mejía (2018, p. 18) afirma que, "la estrategia educativa [de mediadores escolares] para el tratamiento de conflictos, se convierte en el eslabón clave para iniciar el proceso hacia la generación de una cultura para la convivencia pacífica".

Por otra parte, los estudios con relación a la educación en zonas de conflicto y en situación de posconflicto, tales como los desarrollados por Lizarralde (2012), Medrano et al. (20I5), Osorio (20I6), Moreno (20I7), Ramos (20I7), Parra (2017) y Sánchez (2019), vislumbran la necesidad de la construcción de una educación para la paz, en donde la escuela, como institución social, es la llamada a forjar en sus escenarios, herramientas para la resolución pacífica de las controversias surgidas del dinamismo social. Por ende, como lo manifiestan las investigaciones, es imperativo que las instituciones escolares, ubicadas en escenarios de conflicto armado, se vuelvan el eje estructurante de una convivencia pacífica y paralelamente contribuyan a la detención de la destrucción del tejido social, tan afectado por el periodo de violencia en Colombia.

Cabe señalar que no se encontraron estudios publicados en relación con la formación de mediadores escolares en el ciclo complementario de las escuelas normales, siendo estas formadoras de docentes que se desempeñan en escuelas primarias en el territorio colombiano, es pertinente e imperativo el estudio aquí propuesto.

\section{La mediación de conflictos escolares para una educación para la paz}

La frase en latín si vis pacem, para bellum (si quieres la paz, prepárate para la guerra), pareció ser la tendencia dominante con la cual se planteaban las soluciones de los conflictos en la humanidad, inclusive hasta bien entrada la segunda mitad del siglo XX. Este viejo paradigma, gobernó por mucho tiempo el entendimiento de las relaciones conflictuales en la sociedad y está siendo reemplazado por nuevos parámetros de solución de conflicto, no a través de la guerra-violencia, sino con mecanismos alternativos a estas (Ayala, 20I3; Calderón, 2009; Hernández, 2013).

En este sentido, bajo la perspectiva de un estudio que coadyuve al tratamiento de los conflictos en escenarios escolares, la investigación se basó en dos categorías fundamentales, el conflicto (escolar) y la mediación del conflicto, como conceptos estructurantes de una educación para la paz.

Así, entonces, para el entendimiento de la categoría de conflicto, el trabajo acoge los postulados teóricos de Fisas (1998), Lederach $(1998,2000)$ y Galtung $(2003,2004)$, quienes observan el conflicto como la posibilidad de edificación de una paz positiva.

En primera medida, para Fisas (1998), el conflicto, ante todo, es una construcción social, en palabras del autor:

Es una creación humana diferenciada de la violencia (puede haber conflictos sin violencia, aunque no violencia sin conflicto), 
que puede ser positivo o negativo según como se aborde y termine, con posibilidades de ser conducido, transformado y superado (puede convertirse en paz) por las mismas partes, con y sin ayuda de terceros, que afecta a las actitudes y comportamientos de las partes, en el que como resultado se dan disputas; suele ser producto de un antagonismo o una incompatibilidad (inicial, pero superable) entre dos o más partes, el resultado complejo de valoraciones, pulsiones instintivas, afectos, creencias, etc., y que expresa una insatisfacción o desacuerdo sobre cosas diversas. (pp. 185-186)

Bajo ese entendido, el autor plantea que puede presentarse un abordaje positivo o negativo del conflicto, lo cual compartiría Galtung (2003), cuando al referirse a la construcción de paz, plantea que

la paz es el contexto en el cual se despliegan los conflictos de forma no violenta y creativa. Para conocer la paz tenemos que conocer el conflicto y saber cómo pueden transformarse los conflictos, tanto de manera no violenta como de manera creativa. (p. 3I)

Esto se torna imperativo, toda vez que, si no se aprovecha el conflicto como oportunidad para su solución o tratamiento, este puede degradarse en violencia. Al respecto, el autor manifiesta que "los conflictos demandan de nosotros todo lo que tenemos que ofrecer. Si no, las emociones se expresarán fácilmente como violencia, insultando las necesidades básicas de otros como violencia verbal, violencia física o ambas" (2004, p. I5).

La misma postura sería expuesta por Jares (200I), cuando alude que "donde hay vida hay conflicto $y$, además, el conflicto no tiene por qué ser necesariamente negativo" (p. 73). Lo que deja entrever que un escenario conflictivo, máxime si se enmarca en campos escolares, debe ser abordado como una oportunidad/posibilidad de construir armónicamente escenarios de paz para el ser humano.

Al respecto, Lederach aporta con sus estudios que el conflicto puede ser gestionado, transformado y solucionado, pero para ello se debe tener claro que la intervención de este fenómeno debe conducir a la formación de una paz positiva que, a consideración del teórico, es definida de la siguiente manera:

[...] podemos concretar que la paz es la ausencia de condiciones o circunstancias no deseadas (guerras, marginación, hambre, etc.), pero también es la presencia de condiciones y circunstancias deseadas. La paz positiva es la cooperación (la colaboración, la mutua asistencia, el mutuo entendimiento y confianza), es una asociación activa, caracterizada sobre todo por el mutuo beneficio de una relación positiva. (2000, p. 28 )

Por otra parte, en relación con el conflicto en escenarios escolares, siguiendo la línea teórica que se ha explayado, es necesario entenderlo como una posibilidad de mejora de convivencia escolar, toda vez que es en el espacio educativo donde convergen múltiples intereses tanto de estudiantes, docentes, directivos y familias. Por lo tanto, en consideración de Jares (como se citó en Montoya \& Pérez, 2020), el conflicto escolar deber ser encarado y afrontado como un "hecho educativo", de tal manera que se convierta en una oportunidad para un aprendizaje institucional y social.

Aquí es importante entender el papel de la mediación escolar de los conflictos acaecidos en los planteles educativos. En primera medida, Ibarrola e Iriarte (2013) plantean que la mediación escolar es:

un procedimiento para resolver conflictos, pero al mismo tiempo trata de generar en profesores/as y alumnos/as el deseo, los motivos o el impulso necesario para convivir desde la reciprocidad, la cooperación, la responsabilidad social, además de propiciar un buen ambiente de convivencia y recuperar el sentido de comunidad. (p. 368)

Desde este punto de vista, la mediación es entendida como un proceso en el cual un tercero ayuda a la resolución de un conflicto, esta persona ante todo debe ser neutral y ayuda a las partes en disputa de una forma cooperativa y amena para hallar una solución a su controversia (lungman, 1996).

El mismo postulado lo compartiría Habermas (citado por Torres et al., 2015), cuando alude que el diálogo puede convertirse en esa herramienta teórica y práctica para el mejoramiento de la convivencia social.

Por otra parte, Boque (2003) plantea que la mediación "promueve la comprensividad; al aceptar las diferentes versiones de la realidad, defiende la pluralidad; $y$ al fomentar la libre toma de decisiones y compromisos, contribuye a la participación democrática" (p. 10), lo que presupone una colaboración a la tan anhelada cohesión social y las bases para el entendimiento del otro (Rosero, 2019). 
A su vez, la resolución de conflictos escolares trae un plus en el afianzamiento de la democracia, pues a consideración de Fisas (1998) y Lederach (2000), el solo hecho de participación electoral no constituye un ejercicio democrático, sino el fortalecimiento de una sociedad que pueda resolver por ella misma y de manera proactiva conflictos suscitados en la cotidianidad, desde nivel individual, familiar, comunitario e internacional.

Enmarcada en estos planteamientos, esta investigación afirma que es esencial la formación de docentes mediadores de conflictos escolares, que a través de su práctica profesional $y$ en el ejercicio en el aula, puedan determinar las causas de los conflictos presentados al interior de los muros escolares y brindar un tratamiento a las posibles consecuencias que se deriven de estos.

Aunado a lo anterior, es imperativo que los docentes normalistas, que en su mayoría desempeñan su labor en las zonas rurales de Colombia, y que muchas veces actúan en regiones donde ha acontecido o permanece el conflicto armado, tengan herramientas teóricas y prácticas en la solución de los conflictos escolares, concibiendo su papel como sumamente importante en la construcción de una cultura de paz en sus territorios de acción. Esta cultura de paz, tal como lo asevera Fisas (20II), "es una tarea educativa que pasa por educar en y para el conflicto... en llevar a cabo el desarme cultural en la promoción de una ética global y en buscar un consenso fundamental sobre convicciones humanas integradoras" (p. 2).

\section{Metodología}

El presente estudio es de tipo cualitativo, toda vez que el análisis de los sujetos y los fenómenos sociales se debe hacer, según Bernal (2016), de manera holística y contextualizada para una mayor comprensión de la realidad social. Dentro de este trabajo, la investigación cualitativa posibilita abordar con mayor profundidad las vivencias, experiencias e interacciones que tienen los docentes normalistas en formación, con su capacitación en resolución de conflictos escolares.

A su vez, se utilizó el método de estudio de caso, el cual, de acuerdo con Neiman y Quaranta (2009), requiere un análisis profundo de la unidad de trabajo para la comprensión de esta en un sistema integrado al que pertenece y que se está estudiando. Al mismo tiempo, la investigación precisó utilizar el método etnográfico, el cual pretende la descripción de escenarios específicos y se preocupa por analizar las acciones de miembros de una comunidad determinada (Whitehead, 2009).

Para la unidad de análisis, en el año escolar 2019, en el cual se realizó la investigación, se tomó como referente los 1100 estudiantes y 58 docentes de la Escuela Normal Superior del Mayo. Dicha institución ofrece los servicios educativos de preescolar, básica primaria, bachillerato y la formación de ciclo complementario (normalistas), para ejercer la profesión docente. Dentro de los 1100 escolares, 135 se encuentran matriculados a este ciclo de formación complementaria, de los cuales se tomó una muestra de unidad de trabajo de 35 alumnos, quienes estaban inscritos en el cuarto semestre de su formación. Es menester mencionar que se seleccionó a este grupo para la investigación, puesto que era el grupo de licenciados normalistas en formación que, para el momento del estudio, estaban realizando su práctica profesional en distintas escuelas rurales del departamento.

Esta población de unidad de trabajo está compuesta por 35 estudiantes, 17 hombres y 18 mujeres. Sus edades oscilan entre los 18 y 24 años. Los ingresos económicos, recibidos por las familias de los estudiantes, en 31 de los casos, no alcanzan a percibir el salario mínimo mensual vigente, lo que nos lleva a la conclusión de que estamos ante un grupo con precariedad económica, lo que el Departamento Administrativo Nacional de Estadística (DANE, 2013) llamaría "en situación de pobreza". Es preciso mencionar que 28 de los estudiantes viven en la cabecera municipal, el resto vive en la zona rural y en otros municipios como La Unión y San Pablo, en Nariño, y Bolívar en el Cauca, territorios todos atacados por la violencia derivada de los enfrentamientos de grupos al margen de la ley con las fuerzas armadas del Estado.

En una sesión previa al inicio de la investigación se solicitó el permiso al rector de la institución, quien hizo un estudio con docentes y administrativos sobre el proyecto y lo consideraron viable. Posteriormente, hubo una reunión con los estudiantes, junto con el coordinador de los ciclos complementarios de la institución, para informar sobre el objetivo propuesto en el estudio, conseguir el consentimiento informado de cada participante y explicar el tratamiento de la información a la población objeto de estudio.

A esta unidad de trabajo se aplicaron instrumentos de investigación para alcanzar los objetivos propuestos, 
entre ellos, la encuesta, la entrevista semiestructurada y el diario de campo, para identificar las necesidades que se tenían en relación con la formación de mediadores en resolución de conflictos escolares, las cuales fueron procesadas por el software Atlas.ti 8.

Es necesario señalar que las entrevistas y la encuesta, tuvieron como propósito la identificación de las características sociales, económicas y culturales de la población objeto de estudio, además de la identificación de las regiones donde desempeñarían su vida profesional y las herramientas teórico-prácticas que tenían respecto a la resolución de conflictos escolares.

Finalmente, la capacitación brindada a los estudiantes del último semestre del ciclo complementario de la institución educativa consistió en brindar herramientas docentes para la mediación escolar y contó con una serie de talleres teóricos-prácticos para el logro del cometido propuesto. Se realizaron un total de 10 sesiones distribuidas en tres seminarios-talleres, de los cuales se procesó la información en el software Atlas.ti 8.

\section{Resultados}

El ciclo complementario de la Escuela Normal Superior del Mayo: retos hacia la construcción de una educación para la paz

Para este aparte fue necesario analizar dos escenarios para lograr describir los avances que ha tenido la institución con relación a una construcción de una educación para la paz. El primero de ellos, el formal, fue estudiado a través de una comprensión de los principios rectores, el manual de convivencia y el marco legal que obliga a la institución a formar estudiantes en un ambiente sano. Por otro lado, el práctico, correspondiente a las experiencias que ha tenido el plantel educativo respecto a la construcción de paz, para este segundo escenario, se encuestó y entrevistó a docentes de la institución. Es preciso señalar que se hizo énfasis en la formación de normalistas en materia de educación para la paz.

La Escuela Normal Superior de La Cruz contempla en su manual de convivencia dos aspectos importantes sobre la educación para la paz. El primero de ellos es una formación humanista de su estudiantado (Ministerio de Educación Nacional, 2020) y el segundo, un enfoque diferencial, que rige todas las actuaciones tanto administrativas como educativas de la institución. Dicho enfoque, que contempla una perspectiva de género, etnia e inclusión, tiene un enfoque diferencial territorial, el cual:

Consiste en definir acciones a partir de las realidades, de los puntos fuertes y débiles particulares de una zona. La población rural requiere de alternativas educativas pertinentes que tengan en cuenta características geográficas, sociales y culturales, con el fin de acercar la escuela a las comunidades $y$ disminuir la brecha existente entre el campo y la ciudad. De igual manera es necesario que los procesos educativos brinden oportunidades que mejoren la calidad de vida de sus pobladores, contribuyendo a disminuir los índices de violencia y migración a los centros urbanos y desarraigo de sus comunidades, en particular en los establecimientos de Nariño, que en su gran mayoría se ubican en territorios rurales (Institución Educativa Escuela Normal Superior del Mayo, 2017, p. 7).

Dicha orientación es pertinente, toda vez que la institución se encuentra localizada en la zona rural del departamento de Nariño, y además, forma educadores que se desempeñan en gran porcentaje, en escuelas rurales del país.

Por otra parte, la Ley 1620 de Convivencia Escolar (Congreso de Colombia, 20I3) obliga a las instituciones educativas a dar manejo a los conflictos escolares, de tal suerte, que son las responsables de garantizar una convivencia pacífica dentro de estas. En este sentido, el manual de convivencia de la institución, formalmente contempla los MASC, entre ellos la mediación, para cuando se presenten afectaciones en la convivencia dentro del plantel educativo. Asimismo, el Manual de Práctica de los normalistas contempla la necesidad de la formación de una cultura de paz. Lo anterior tiene el propósito, tal como lo afirman Loaiza (20II) y Muñoz (20I5), de formar maestros que aporten al mejoramiento de una cultura pedagógica en el país, tanto en el nivel teórico, como en el investigativo y práctico.

Con esto, la institución en el escenario práctico, ha formulado un proyecto pedagógico en educación para la paz y la democracia, dirigido por docentes del área de ciencias sociales; dicho proyecto tiene como cometido el fomento en todos los grados, de una cultura democrática a través de la elección de representantes estudiantiles y la formación de valores cívicos y cultura ciudadana (Quintero, 2020). Sin embargo, con esto no se suplen las necesidades de la formación docente normalista, en el entendido de que estos precisan de herramientas pertinentes para la 
intervención, gestión y resolución de conflictos escolares en su ejercicio profesional.

Por otra parte, respecto a la formación de elementos teóricos e históricos sobre el conflicto armado en Colombia y su incidencia en su vida profesional, un estudiante del ciclo complementario de la institución afirma lo siguiente:

Si bien el colegio se ha enfocado en dictarnos la historia del conflicto y posconflicto en Colombia, en la materia de ciencias sociales, nosotros como estudiantes vemos la necesidad de formarnos en elementos de cómo abordar dicha problemática en las instituciones donde nos desempeñamos. Además, dicha formación histórica solo llega hasta el grado $\mathrm{I} I$, en nuestro ciclo complementario no la tenemos, y yo la veo necesaria, porque la mayoría de nosotros, los que seguimos la docencia, nos desempeñamos en la mayoría de veces en zonas rurales con presencia de conflicto. (Comunicación personal, Guerrero, 2019)

Sin embargo, es preciso señalar que específicamente en el ciclo complementario se vienen adelantando procesos de educación para la paz, concernientes en el fortalecimiento de la cultura de paz a través de competencias ciudadanas y la participación democrática, enseñando a los docentes normalistas elementos didácticos y pedagógicos sobre el fortalecimiento de una ciudadanía mucho más cívica y más participativa (Comunicación personal, Imbachí, 2019).

Por último, según encuesta realizada en esta investigación a los 35 estudiantes objeto de estudio, 30 de estos afirman que ven necesaria la capacitación en resolución de conflictos, puesto que su formación en esta temática ha sido nula. Resultados parecidos arrojaría la encuesta aplicada a 54 docentes de la institución, entre los cuales, 37 aluden que no conocen un procedimiento asertivo para resolver un conflicto dentro de la institución educativa. Esto devela que los docentes que forman docentes no tienen las herramientas suficientes para abordar esta problemática.

Este panorama, que parece desalentador, es visto como una oportunidad para el mejoramiento institucional, toda vez que proyectos como el presente, y previos, desarrollados en el fortalecimiento de una cultura de paz, demuestran el interés del plantel educativo en apuntar a convertirse en una institución fomentadora de paz en escenarios escolares.
Lineamientos para la formación de mediadores en zonas de conflicto armado

El propósito de esta investigación es establecer lineamientos para la formación de mediadores escolares en zonas de conflicto armado, realizando un estudio de caso con los normalistas de la Escuela Normal Superior del Mayo del municipio de La Cruz, en el departamento de Nariño.

En un primer momento, al indagar sobre los proyectos profesionales de las 35 personas objeto de estudio, se observa que 15 estudiantes afirman querer continuar con su formación profesional estudiando alguna carrera universitaria, de preferencia una licenciatura; 20 planean ejercer como docentes normalistas en las instituciones educativas.

Asimismo, al preguntar sobre las zonas donde proyectan ejercer su vida profesional, los estudiantes aluden que se desempeñarán en zonas rurales del territorio nacional, pero en gran medida, en municipios como Bolívar, Corinto y Toribío, en el departamento del Cauca, y municipios como La Unión, La Cruz, San Bernardo, San Pablo, Policarpa, Leiva y Cumbitara, en el departamento de $\mathrm{Na}$ riño, zonas atacadas por el conflicto armado.

El coordinador del ciclo complementario de la institución, Gerardo Imbachí, concuerda con lo anterior, cuando afirma, basado en un estudio de seguimiento a egresados, que los docentes normalistas formados en la institución ejercen en escuelas multigrados, de zonas rurales del país, en departamentos del Cauca, Nariño y Putumayo, preferiblemente (2019).

Por otra parte, al averiguar sobre su práctica pedagógica, como requisito de grado para el año 2019 , todos los estudiantes la están realizando en veredas del municipio de La Cruz y otras zonas del departamento de Nariño.

Aunado a lo anterior, los 35 estudiantes aseguraron no tener una formación en resolución de conflictos escolares y que en el momento de la investigación no tenían conocimiento de cómo actuar al presentarse un problema de violencia escolar.

Es importante señalar que al actuar en zonas rurales y especialmente en escuelas multigrados, los docentes normalistas se encuentran solos en su lugar de trabajo, $y$ al ser un maestro en estos lejanos territorios, culturalmente 
Tabla I. Conceptos de conflicto, violencia escolar y paz del cuarto semestre del ciclo complementario

\begin{tabular}{|c|c|c|}
\hline Concepto & Idea previa & Idea poscapacitación \\
\hline Conflicto & $\begin{array}{l}\text { "Es una problemática que se presenta entre personas } \\
\text { que no saben dialogar". }\end{array}$ & $\begin{array}{l}\text { "Situación social, en la cual, las partes tienen una disputa } \\
\text { por intereses opuestos". }\end{array}$ \\
\hline Violencia escolar & $\begin{array}{l}\text { "Es cuando los niños y niñas se agreden física y verbal- } \\
\text { mente dentro de la institución". }\end{array}$ & $\begin{array}{l}\text { "Es la degradación de un conflicto, cuando se usa la fuerza, } \\
\text { sea física o psicológica y verbal, para ejercer daño a una } \\
\text { persona dentro de las instalaciones educativas". }\end{array}$ \\
\hline $\mathrm{Paz}$ & $\begin{array}{l}\text { "Se la puede percibir como una cierta tranquilidad, donde } \\
\text { no haya guerras". }\end{array}$ & $\begin{array}{l}\text { "Se la podría definir a través una situación social con } \\
\text { un alto grado de efectividad de derechos fundamentales } \\
\text { y un alto grado de justicia social". }\end{array}$ \\
\hline
\end{tabular}

Nota: recogida del taller de un estudiante del ciclo complementario y datos arrojados del procesamiento en el software Atlas.ti 8

se convierten en un referente de respeto y poder de la comunidad.

Por lo tanto, se exponen aquí los lineamientos básicos para la formación de mediadores escolares, acogidos también por teóricos como Mejía (20I8), como una competencia necesaria que deben tener los docentes para la construcción de una cultura de paz y sana convivencia, tanto en sus instituciones educativas como en sus territorios.

\section{Primer seminario taller: Conociendo el concepto de} conflicto y aprendiendo sobre el conflicto armado Esta fase de capacitación teórica se desarrolló en cuatro sesiones. Las dos primeras se desenvolvieron con base en las preguntas ¿qué es el conflicto?, ¿qué es violencia?, ¿qué es conflicto escolar?, ¿qué es violencia escolar?, ¿qué es la paz?; aquí se presentaron de manera general teorías sobre los conceptos. A la vez se analizaron la violencia, sus causas (internas y externas) y la función de la escuela en la construcción de una cultura de paz.

También se estudiaron las dimensiones del conflicto (su grado de intensidad, de conciencia y de pureza), la estructura del conflicto (partes, problemas y proceso), la dinámica del conflicto (formación, intensificación, escalada y transformación).

Antes de la capacitación se realizó un ejercicio de lluvia de ideas sobre los conceptos mencionados, en el que cada estudiante señalaba en una hoja de papel lo que pensaba sobre las palabras conflicto, violencia escolar y paz; al finalizar la fase teórica sobre estos se volvió a aplicar el ejercicio sobre los conceptos, en la misma hoja de papel, para hacer un contraste sobre la formulación conceptual de dichos términos, lo que después de un procesamiento de datos en Atlas.ti 8 en un panorama general, encontró lo siguiente (ver tabla I).
Se observa que, después de la capacitación, los estudiantes formulan definiciones mejor elaboradas, basados en teorías y conocimiento previo. Esto es fundamental para el entendimiento de las dinámicas del conflicto y de los fenómenos en los cuales se verán sumergidos, tales como la violencia escolar.

Las otras dos sesiones consistieron en el refuerzo de los conocimientos históricos del conflicto armado y su incidencia en las relaciones sociales, económicas y culturales en las zonas afectadas. Asimismo, se pidió a los estudiantes elaborar una reseña histórica de los territorios en los cuales estaban desempeñando su práctica profesional, con el propósito de adquirir una contextualización integral de las localidades donde trabajan. Se solicitó igualmente, que elaboraran una lista con los hechos victimizantes que sufrieron las poblaciones donde se desempeñaban como docentes practicantes; entre los más frecuentes se encuentran los que se presentan en la tabla 2 .

Tabla 2. Hechos victimizantes con mayor frecuencia en las zonas donde desempeñan los normalistas en formación

\begin{tabular}{lc} 
Hecho victimizante & Número de reseñas en la que aparece \\
Homicidio & $30 / 35$ \\
$\begin{array}{l}\text { Desplazamiento } \\
\text { forzado }\end{array}$ & $28 / 35$ \\
Despojo de tierras & $26 / 35$ \\
Amenazas & $21 / 35$ \\
\hline
\end{tabular}

Nota: recogida de las reseñas y lista elaborada por los 35 estudiantes, procesado por Atlas.ti 8

Lo anterior pone en evidencia la necesidad de aplicar la competencia de sensibilidad social, contemplada en el Pacto de Práctica 2017-2023, de la Escuela Normal Superior del Mayo, que obliga al docente normalista a una observancia analítica y comprensiva de su escenario de acción. Postulado que constata la futura docente normalista, Bolaños en su comunicación personal, cuando dice que: 
la importancia de conocer la historia y los hechos de conflicto armado de los territorios donde nos desempeñamos como docentes, es trascendental, ya que nos ayuda a entender que los niños y niñas con los cuales trabajamos, padecen una problemática social muy fuerte y si se junta con la violencia escolar, se torna algo muy grave, por lo cual, es nuestro deber, entender y comprender para luego garantizar un ambiente más pacífico por lo menos en las escuelas. (Comunicación personal, 2019)

Segundo seminario taller: Elementos teóricos de la resolución de conflictos y el diagnóstico del conflicto escolar

Este taller se trabajó mediante dos sesiones. El objetivo primordial es que los futuros docentes normalistas tengan herramientas necesarias para la identificación de conflictos escolares y el tratamiento, gestión y resolución alternativa de estos.

Para ello, en la primera sesión se abordaron los elementos teóricos de la resolución de conflictos, y se afianzó el conocimiento teórico de la construcción de una paz positiva, tomando como referentes a Galtung $(2003,2004)$, Fisas $(1998,201 \mathrm{I})$ y Lederach $(1998,2000)$.

La segunda sesión tuvo el objetivo de brindar herramientas sobre cómo realizar un diagnóstico del conflicto escolar (e.g., ver figura I). Para ello se realizó la metodología de "el árbol de problemas", en la que los estudiantes realizaron una identificación de casos repetitivos (ubicados debajo del problema central) de una raíz central que es la violencia escolar dentro de su institución educativa; después, buscaron las causas de los respectivos casos (ubicados debajo de los casos) y finalmente expresaron las manifestaciones (exteriorización del conflicto) más comunes de los problemas.

Para lo anterior, los 35 estudiantes se dividieron en siete grupos de cinco integrantes. Cada grupo identificaba los casos, sus causas y sus respectivas manifestaciones. Entre los casos más comunes que se desprenden de los grupos, podemos afirmar que son: drogadicción, indisciplina (agresiones verbales e irrespeto hacia docentes) y suicidio. Entre las causas más frecuentes se encuentran el desempleo, la desintegración familiar, la crisis económica y el conflicto armado. Entre las manifestaciones más recurrentes se encontraron el hurto, la agresividad, los insultos a compañeros y profesores, el bajo rendimiento escolar y la deserción estudiantil.
Figura I. Extraída del texto "Resolución de conflictos y convivencia escolar" (Mejía, 2018)

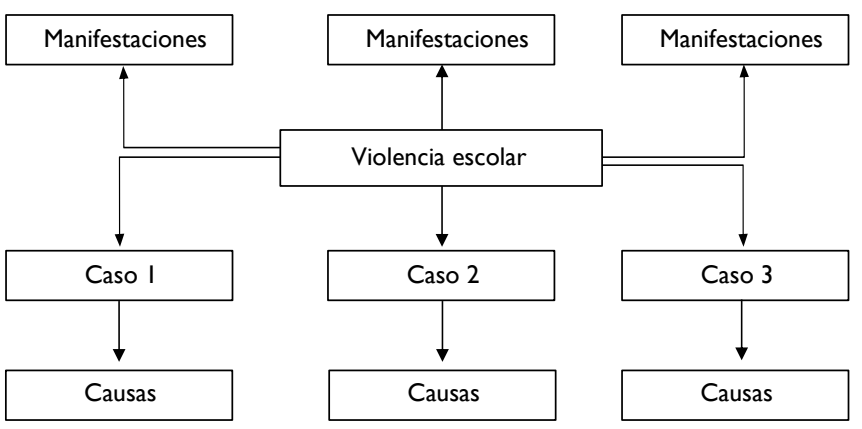

Por otra parte, para el diagnóstico del conflicto escolar se explicó la herramienta de la "escucha activa", metodología compuesta por ocho pasos a saber: (I) mirar a la persona con quien estamos hablando, (2) poner atención a lo que está diciendo, (3) escuchar sin interrumpir hasta que termine de hablar, (4) tratar de entender el punto de vista de la otra persona, (5) poner atención al lenguaje corporal $y$ al tono de voz de la persona, buscar pistas para saber lo que siente, (6) hacer preguntas para aclarar y promover entendimiento, (7) parafrasear lo que acaba de escuchar en forma modificada con sus propias palabras y (8) escuchar reflexivamente, reconocer la importancia de los sentimientos de esa persona (Mejía, 2018, p. 55).

Con esto, los docentes, a través de una comunicación asertiva, pueden comprobar la gravedad de los asuntos a los que se enfrentan en las instituciones educativas $y$, a su vez, generan lazos de confianza con la comunidad educativa.

Tercer seminario taller: Métodos alternativos de solución de conflictos. La mediación del conflicto escolar

Los MASC nacen con la intención de terminar y transformar el conflicto, para que este no resulte en violencia. Precisamente son alternativos porque no usan la violencia física para resolverlos, ni tampoco ponen en movimiento el aparato jurisdiccional del Estado para su respectiva solución.

Por ello, este taller consistió en dos fases de cuatro sesiones. Las dos primeras tenían el cometido de dar a conocer lo siguiente: ¿qué son los MASC?, ¿cuáles son sus beneficios?, ¿cuáles son los más comunes (negociación, comunicación asertiva, mediación y arbitraje)?, ¿en qué consiste la mediación?, ¿cuál es el perfil de un mediador (sensibilidad social, asertivo, neutral)? y ipor qué la mediación 
es el método más útil para los conflictos al interior de las instituciones educativas?

La tercera sesión consistió en hacer ejercicios prácticos de preparación de mediadores, en donde los docentes normalistas en formación, separados en grupos de tres integrantes, simulaban una escena de mediación, con un caso cotidiano de conflicto escolar, en donde dos de ellos eran las partes de la pugna y uno el mediador. Después, se solicitó a los estudiantes del cuarto semestre del ciclo complementario de la Escuela Normal Superior del Mayo, que implementaran lo aprendido en su práctica pedagógica.

También, se exhorta a la población objeto de estudio a que dicha formación la deben aplicar en sus lugares de trabajo, para que ellos, como docentes, también formen mediadores escolares en las instituciones educativas, en especial, estudiantes, para que estos últimos tengan la base de un ejercicio de ciudadanía activa.

Finalmente, la cuarta sesión, llevada a cabo tres meses después de la tercera, tuvo como objetivo constatar si lo aprendido tuvo efectos positivos en el ejercicio de la práctica pedagógica de los futuros docentes normalistas. En efecto, según anotaciones del diario de campo de la investigación, se observó que el grupo de trabajo muestra mejoras sustanciales en el tratamiento de los conflictos escolares, pues se pasó de frases como "no sé actuar cuando se presentan situaciones de violencia en mi aula de clase" a "después del conocimiento de los MASC, me siento preparado para afrontar los conflictos en mi vida profesional". Esto es respaldado con la encuesta final que se realizó a los 35 estudiantes objeto de estudio, los cuales, al formularles la pregunta: ¿considera que después de la capacitación en mediación de conflictos escolares usted aborda el conflicto de una manera más proactiva?, 33 de ellos contestaron afirmativamente.

\section{Discusión}

El fenómeno social de la educación, estudiada desde teóricos sociológicos clásicos como Azevedo (1942) compone un proceso de formación constante, de manera tal que abarque una concepción integral del ser humano, toda vez que:

el fin central de la educación es posibilitar el pleno desarrollo de las personas y el desarrollo individual y colectivo de las personas, los cuales deben alcanzarse en interacciones directas con el contexto social al que pertenecen. Para su logro, se deben cimentar derechos humanos como el respeto por la vida, la paz, los principios democráticos y de convivencia, el pluralismo, la justicia, la solidaridad, la tolerancia y la libertad. (Soler, 20 I I, p. I2)

Desde este punto de vista, se debe dejar la función reduccionista de la institución escolar, la cual es la simple transmisión de conocimientos, y pensar dicha institución como el escenario donde se estructuran las relaciones sociales entre estudiantes, las cuales deben organizarse para contribuir a la formación de una paz estable.

En este sentido, afirmaría Fisas (1998) que "el proyecto de construir una cultura de paz en buena medida no es otra cosa que el reto planetario de abordar los conflictos desde otra mirada, con otros utensilios y otros propósitos" (p. 183). En este sentido, se vuelve pertinente la elaboración de proyectos que tengan como objetivo formar docentes para la resolución pacífica de conflictos en escenarios escolares, toda vez que los educadores se convierten, dentro de sus instituciones, en un referente importante para la solución de las problemáticas escolares.

Dicha actividad debe contemplar una docencia que aborde los conflictos de una manera compleja e integral, pues como lo advertiría Moreno (2017), se debe llevar a:

[...] plantear la práctica docente en torno a una pedagogía problematizadora, en la que el docente articule sus problemas en la práctica, con los problemas sociales que envuelven a sus estudiantes $y$ las intencionalidades de construir, como ordena la ley, una cultura proclive para la paz. Esto envuelve la búsqueda profunda de las "causas” que históricamente han determinado dichos problemas (p. 137).

Lo anterior es fundamental para el caso concreto, toda vez que, como se afirmó en la investigación, la formación de mediadores escolares en zonas de conflicto armado debe examinar, de manera inexorable, las condiciones históricas, sociales, culturales, políticas y económicas, que este fenómeno ha configurado en los territorios en donde se ha suscitado.

Además de lo anterior, la formación de docentes normalistas en el marco de la resolución de conflictos escolares, brinda un apoyo al mejoramiento de la calidad de profesores, puesto que, actualmente, con mandato legal, los maestros deben contar con herramientas teórico-prácticas 
para la construcción de una convivencia más pacífica dentro de los planteles educativos. Actividad que se torna de gran importancia para los docentes normalistas, debido a que, cuando ejercen su profesión, lo hacen en zonas rurales, muchas veces en escuelas unitarias, en donde no existe otra autoridad administrativa y educativa más que el docente.

Por esa razón, en las escuelas rurales y los pueblos apartados del casco urbano, estos profesores todavía cumplen un papel destacado en la educación. Además, porque en estas zonas hacen falta profesores. Según la revista Dinero, en el campo, el $51 \%$ de las sedes educativas son multigrado (escuelas que tienen un profesor para varios grados).

De ahí la necesidad de tener más profesores de calidad en las zonas rurales y la relevancia que tomaron las normales superiores, de las cuales el $90 \%$ están en poblaciones con menos de 100000 habitantes.

Lo cierto es que la formación de maestros es un aspecto fundamental para mejorar el desarrollo de estas regiones y para implementar la construcción de paz en los municipios que más sufrieron con el conflicto, un asunto particularmente relevante en este momento. (Revista Semana, 2017, párrs. 2-3)

Lo anterior es importante si se considera que las escuelas normales, tal como la ubicada en el municipio de La Cruz, en el departamento de Nariño, forman maestros para la educación preescolar y la básica primaria, niveles educativos claves para cimentar las bases de una cultura democrática y pacífica (MEN, 20I5b).

En esta medida, los docentes normalistas en formación son conscientes de su papel fundamental en la resolución de conflictos escolares y consideran que el diálogo, como mecanismo que lleva a la mediación de los conflictos, es la manera más democrática, pacífica y alternativa de tratar dichos problemas. Es así, entonces, que los lineamientos presentados para la capacitación de mediadores escolares contempla la necesidad de una formación integral, holística y compleja, en la que el docente tenga una competencia de sensibilidad social, una competencia histórica $y$, ante todo, una competencia educativa que le permita la comprensión, con mayor profundidad, de las realidades que rodean su vida personal y profesional.

Finalmente, y siguiendo la línea teórica de Galtung $(2003,2004)$, Fisas $(1998,201 \mathrm{I})$ y Lederach $(1998,2000)$. entendemos que el conflicto, y sobre todo el conflicto escolar, es parte de la esencia de la vida educativa. Bajo ese parámetro, es necesario enmarcar al conflicto como la posibilidad y oportunidad de construir, con base en este, una paz positiva, la cual cimiente desde la juventud, los principios democráticos de una sana convivencia, que si se inculca en las instituciones educativas puede transmitirla a la sociedad en general y puede procurar el respeto, la comprensión del otro y la necesidad de convivir en la diferencia y la diversidad.

\section{Conclusiones}

En primera medida es imperativo afirmar que las instituciones educativas desempeñan un papel fundamental en la construcción de una cultura de paz positiva, puesto que estas tienen la función y deber de brindar un ambiente de sana convivencia a toda la comunidad educativa.

Por otra parte, las escuelas normales superiores, en especial la Escuela Normal Superior del Mayo, ubicada en el municipio de La Cruz, departamento de Nariño, deben contemplar en sus currículos, como eje transversal, la formación de docentes mediadores de conflictos escolares, toda vez que con dicha instrucción, se brindan herramientas teórico-prácticas a los futuros docentes de preescolar y básica primaria de las zonas rurales del país, procurando el mejoramiento de la calidad educativa y un ambiente más sano.

Asimismo, la capacitación de estos docentes, que en su mayoría ejercerán su profesión en territorios afectados por el conflicto armado, debe contemplar la necesidad de formar competencias de sensibilidad social y comunicación asertiva y, a la vez, que los estudiantes adquieran un conocimiento histórico de dicho fenómeno. Cabe señalar que es menester la materialización del enfoque diferencial territorial en dicha formación, en el entendido de que este esquema ayudará a una mejor comprensión de la realidad social que enfrentan tanto docentes como estudiantes.

Lo anterior se torna especialmente relevante cuando se contempla la idea de que el docente normalista, que se desempeña en escuelas unitarias o multigrados, por lo general se encuentra solo en la institución educativa, lo que lo hace un referente de autoridad y respeto dentro de la comunidad en la que actúa. 
Finalmente, se puede afirmar que los docentes normalistas son convocados a dos cosas esencialmente. La primera, mitigar y solucionar el conflicto escolar que se le presente en el plantel educativo, y la segunda, derivada de la primera, es que una vez dado el tratamiento respectivo al conflicto escolar, que también es social, el docente previene que se dañe aún más el tejido social de comunidades afectadas por el conflicto armado.

\section{Conflicto de intereses}

Artículo derivado del programa Jóvenes Investigadores e Innovadores Colciencias-Universidad de Nariño, convocatoria número 812-2018. Asesora: Carmen Patricia Cerón Rengifo.

\section{Referencias}

Alcaldía Municipal de La Cruz (2016). Plan de desarrollo municipal 2016-2019. Alcaldía de La Cruz.

Alcaldía Municipal de La Cruz (2020). Plan de desarrollo municipal 2020-2023. Alcaldía municipal de La Cruz.

Ayala, G. (20|3). Análisis del conflicto y la mediación como método de resolución: Redorta y Moore. Revista Academia y Derecho, 4(7), 79- 106.

Azevedo, F. (1942). Sociología de la educación. Introducción al estudio de los fenómenos pedagógicos y de sus relaciones con los demás fenómenos sociales. Fondo de Cultura Económica.

Bastidas, M., \& Ordóñez, S. (2004). Violencia y conflicto armado: una reconstrucción sociológica del municipio de La Cruz 19982002. Universidad de Nariño.

Benavides, M. (2013). Tipología de la conflictividad escolar en las instituciones educativas del municipio de Pasto. Universidad de Nariño.

Bernal, C. (2016). Metodología de la investigación. Pearsons.

Boque, M. (2003). Cultura de mediación y cambio social. Gedisa.

Calderón, P. (2009). Teoría de conflictos de Johan Galtung. Revista Paz y Conflictos, 2, 60-81. http://redcimas.org/wordpress/wp-content/uploads $/ 2012 / 08 / \mathrm{m} \_$Galtung_LAteoria.pdf

Chaux, E. (2012). Educación, convivencia y agresión escolar. Ediciones Uniandes.

Departamento Administrativo Nacional de Estadística (2013). Pobreza en Colombia. https://www.dane.gov.co/files/investigaciones/condiciones_vida/pobreza/cp_pobreza_20122.pdf
Fernández, J., \& Galán, J. (2010). Caso de mediación escolar, formación de mediadores escolares: un proyecto en expansión. Revista de Mediación, 3(6), 50-55. https://revistademediacion.com/wp-content/uploads/2013/08/RevistaMediacion-6-07.pdf

Fiorino,V., Fernández, L., Fernández, S., Ferreiro, C., \& Villar, O. (2019). Conflicto, convivencia y cultura de paz. La resolución de conflictos en ámbito educativo como preparación para la paz. Revista de Cultura de Paz, 3, 335-349.

Fisas,V. (1998). Cultura de paz y gestión de conflictos. Icaria.

Fisas, V. (20I I). Educar para una cultura de paz. Quaderns de Construcció de Pau, 20(I), 2-10.

Galtung, J. (2003). Paz por medios pacíficos. Paz y conflicto, desarrollo y civilización. Gernika Gogoratuz.

Galtung, J. (2004). Trascender y transformar: una introducción al trabajo de conflictos (F. Montiel, Trad.). Quimera.

Guajardo, N. (2010). Resolución de conflictos y clima escolar:la formación permanente del profesorado en resolución de conflictos. Revista Temas, 91-102.

Hernández, E. (20I3). Mediaciones en el conflicto armado colombiano. Hallazgos desde la investigación para la paz. Confines de Relaciones Internacionales y Ciencia Política, 9(18), I-32.

Ibarrola, S., \& Iriarte, C. (20|3). La influencia positiva de la mediación escolar en la mejora de la calidad docente e institucional: percepciones del profesor mediador. Profesorado: Revista de Currículum y Formación de Profesorado, I 7(I), 367-384.

Institución Educativa Escuela Normal Superior del Mayo. (30 de enero de 2017). Manual de Convivencia. Un proyecto de vida normalista. Institución Educativa Escuela Normal Superior del Mayo.

lungman, S. (1996). La mediación escolar. Lugar Editorial. http:// www.terras.edu.ar/biblioteca//6/16TUT_lungman_Unidad_3.pdf

Jares, X. (200I). Conflictos y experiencias de educación para la convivencia en el ámbito escolar. 12 Jornadas de Consejos Escolares de las Comunidades Autónomas y del Estado (pp. 71-99). Consejo Escolar de Galicia.

Lederach,J. (1992). Enredos, pleitos y problemas. Ediciones ClaraSemilla.

Lederach, J. (2000). El abecé de la paz y los conflictos. Catarata.

Lederach, J. P. (1998). Construyendo la paz. Reconciliación sostenible en sociedades divididas. Bakeaz.

Lizarralde, M. (2012). Ambientes educativos y territorios del miedo en medio del conflicto armado: estudio sobre escuelas del Bajo y Alto Putumayo. Revista Colombiana de Educación (62), 2I-39. 
Loaiza, Y. (20I I). Las escuelas normales superiores colombianas: reformas y tensiones en la segunda mitad del siglo $X X$. Revista Latinoamericana de Estudios Educativos, 7(2), 67-93.

Luna, N. (2018). La mediación escolar como estrategia para la convivencia en la escuela. La mediación escolar como estrategia para la convivencia en la escuela (Tesis de grado). Pontificia Universidad Javeriana, Cali (Colombia). http://vitela.javerianacali.edu.co/handle/ I I522//0776

Margolin, G., \& Ceballos, M. (2004). Bogotá: anatomía de una transformación. Políticas de seguridad ciudadana 1995-2003. Pontificia Universidad Javeriana.

Martínez, M. (2018). La formación en convivencia: papel de la mediación en la solución de conflictos. Educación y Humanismo, 20(35), I27-142. https://doi.org//0.1708//eduhum.20.35.2838

Medrano, O., Ochoa, W., \& Quiroga, L. (2015). La educación en situación de conflicto armado y postconflicto: aprendizajes para Colombia a partir de los casos de Argentina, El Salvador, Guatemala y Sudáfrica (Tesis de maestría). Universidad Santo Tomás, Bogotá (Colombia).

Mejía, J. (2018). Resolución de conflictos y convivencia escolar. Universidad de Nariño.

Ministerio de Educación Nacional (2015a). La educación en cifras. Ministerio de Educación de Colombia.

Ministerio de Educación Nacional (2015b). Naturaleza y retos de las escuelas normales superiores. Ministerio de Educación de Colombia.

Ministerio de Educación Nacional (20 de mayo de 2020). Perfil del normalista superior. http://aprende.colombiaaprende.edu.co/ckfinder/userfiles/files/Sobre_el_perfil_del_ normalista_superior.pdf

Montoya, D., \& Pérez, S. (2020). La lúdica como estrategia didáctica para la mediación del conflicto escolar en el $6^{\circ}$ de la Institución Educativa Cristóbal Colón de Armenia, Quindío. Plumilla Educativa, 25(I), 99-123.

Moreno, E. (2017). Educación, conflicto y postconflicto en Colombia. Revista Diálogos de Saberes, 46, I25-142.

Muñoz, J. (2015). Legado educativo de las escuelas normales en la formación de maestros superiores en Colombia. Revista Criterios, 22(I), 183-197.

Neiman, G., \& Quaranta, G. (2009). Los estudios de caso en la investigación sociológica. En I.Vasilachis (Ed.), Estrategias de investigación cualitativa (pp. 2/3-2/7). Gedisa.

Organización de las Naciones Unidas (2019). Behind the numbers: Ending school violence and bullying. Unesco.

Organización de las Naciones Unidas para la Educación, Ciencia y Cultura (I de octubre de 2018). Nuevos datos revelan que en el mundo uno de cada tres adolescentes sufre acoso escolar. https://es.unesco.org/news/nuevos-datos-revelanque-mundo-cada-tres-adolescentes-sufre-acoso-escolar
Ortega, D. (12 de septiembre de 20I2). Mecanismos de resolución de conflictos en la Institución Educativa Municipal Antonio Nariño. Universidad de Nariño.

Ortega, R. (2010). Agresividad injustificada, bullying y violencia escolar. Alianza.

Osorio, J. (2016). La escuela en escenarios de conflicto: daños y desafíos. Hallazgos, 13(26), 179-191. https://doi. org/I0.15332/s I794-384I.2016.0026.07

Pardo, M. (2019). El derecho a la vida y a la paz le son propios a la escuela. En J. Ávila, O. Rojas, L. Escobar, L. Cañón, J. Restrepo, H. Sánchez, ... J. Ramírez (Eds.), La escuela territorio de paz (pp. 9-II). Publicaciones CEID-Fecode.

Parra, A. (2017). Violencia escolar: prevención y mediación en la esfera del posconflicto colombiano. En M. Prieto (Ed.), Investigaciones y propuestas de intervención sobre violencia escolar en Iberoamérica (pp. |3-6I). Universidad de Guadalajara.

Pérez, M., Gázquez, J., Morelo, M., Martos, Á., Simón, M., \& Barragán, A. (2016). La convivencia escolar: un acercamiento multidisciplinar. Asunivep.

PNUD (20I I). Informe sobre desarrollo humano. Ciudad de México: Ediciones Mundi Prensa.

Quintero, D. (2019). Configuración de preferencias electorales en el municipio de La Cruz-Nariño, en torno al plebiscito por la Paz en Colombia. Flacso-Ecuador.

Quintero, D. (2020). Formación docente en educación para la paz en la Escuela Normal Superior del municipio de La Cruz-Nariño. Artículo inédito sin publicar, I-22.

Ramos, J. (2017). Enseñanza y aprendizaje del conflicto armado en Colombia: prácticas docentes y conocimiento escolar. Universidad Autónoma de Barcelona (Tesis de doctorado). Universitat Autònoma de Barcelona, Barcelona (España). https:// www.tdx.cat/handle/ I0803/458020\#page $=$ I

Revista Semana (17 de agosto de 2017). iLas escuelas normales están en vía de extinción? Revista Semana. https://www. semana.com/educacion/articulo/formacion-de-maestrosla-importancia-de-las-escuelas-normales/536758

Rosero, J. (2019). El objetivo de la educación desde el texto "La pedagógica latinoamericana". Revista Unimar, 37(2), 18I187. https://doi.org/10.31948/Rev.unimar/37-2-art10

Salcedo,A., \& Jennings,Y. (2016). La mediación como herramienta de resolución de conflictos en el sistema educativo dominicano. Ministerio de Educación República Dominicana.

Sánchez, H. (2019). Las escuelas en paz y para la paz sí son posibles. En J. Ávila, O. Rojas, L. Escobar, L. Cañón, J. Restrepo, H. Sánchez, ... J. Ramírez (Eds.), La escuela territorio de paz (pp. 95-102). Publicaciones Ceid-Fecode. 
Santiesteban,A. (2004). Formación de la ciudadanía y educación política. En M. Vera, \& D. Pérez (Eds.), La formación de la ciudadanía (pp. 377-388). Universidad de Alicante.

Soler, J. (201 I). El desarrollo de competencias ciudadanas en la escuela. Módulo: "la mediación de conflictos entre pares y la formación de competencias ciudadanas en la escuela". Opciones Gráficas Editores.

Torres, J., Galván, G., \& Hernández, O. (2015). Ética del discurso de Habermas: como propuesta teórico-práctica en la solución de problemas práctico-morales. Justicia, 29, 13-27.

Vivias, J. (2I de febrero de 2018). Matoneo, problema que no se ha podido erradicar de colegios del país. ElTiempo. https:// www.eltiempo.com/colombia/otras-ciudades/matoneoen-las-ciudades-de-colombia-185084
Whitehead, K. (2019). Using ethnomethodology and conversation analysis to study social categories: The case of racial categories in South African radio talk. En S. Laher,A. Fynn, \& S. Kramer (Eds.), Transforming Research Methods in the Social Sciences (pp. 25I-264).Wits University Press.

\section{Referencias legales}

Congreso de Colombia (2013). Ley de Convivencia Escolar ( 1620 del I5 de marzo de 2013). Por la cual se crea el Sistema Nacional de Convivencia Escolar y formación para el ejercicio de los derechos humanos, la educación para la sexualidad y la prevención y mitigación de la violencia escolar. 